\title{
DIBUJO Y REPETICIÓN: PRESENCIA MANUAL DE ENRIC MIRALLES EN LA PLANTA DEL AYUNTAMIENTO DE UTRECHT
}

\author{
Javier Fernández Contreras
}

\begin{abstract}
En el archivo de la Fundación Miralles en Barcelona se conservan 479 dibujos originales de Enric Miralles para el proyecto del Ayuntamiento de Utrecht. La mayoría son croquis y planos de planta elaborados en papel vegetal, cuyo tamaño oscila aproximadamente entre el DIN A5 y el DIN A3, principalmente en escalas 1/400 y 1/200. Este artículo explica la evolución del proyecto desde los dibujos de Miralles, ilustrando cómo en la repetición y seriación de dicho proceso se produce una incorporación de su manera de dibujar a la geometría de la planta del edificio original.
\end{abstract}

Palabras clave: Miralles, dibujo, repetición, planta, Utrecht

Keywords: Miralles, Drawing, Repetition, Floor Plan, Utrecht

\begin{abstract}
"En el solar había una serie de típicas casas profundas holandesas de distintas épocas, que se cerraban. Y, con el paso de los años, la más importante, que tenía un patio, se unió con dos o tres vecinas y se transformó en el Ayuntamiento. Luego se fueron añadiendo las siguientes. Más tarde llegó un arquitecto neoclásico, quien -yo creo que con poca habilidad-construyó el patio central, inventando una fachada simétrica cuyos ejes no se correspondían con el patio, que es la cosa menos clásica del mundo".

"Todavía estoy completamente involucrado en el proceso. Todavía dibujo mucho. Trato de dibujar las cosas por mí mismo en la medida que puedo. La gente del estudio todavía tiene gran confianza en mis planos, que son bastante precisos. Trato de ir más allá de hacer simples croquis, sigo dibujando por mí mismo y configuro así la geometría. No me gusta pensar que los proyectos son sólo el resultado de croquis, y no me conformo con hacer un esquema básico y pasárselo a otra persona. Siempre he trabajado en formatos pequeños porque para mí es más fácil dibujar así, pero voy más allá del simple croquis"².
\end{abstract}

Enric Miralles. 1999

Cuando, en febrero de 1997, Enric Miralles y Benedetta Tagliabue se presentan al concurso para la remodelación del Ayuntamiento de Utrecht, se enfrentan a un edificio que lleva construyéndose siglos, primero como un conjunto de casas medievales vinculadas a las actividades comerciales del Oudegracht -canal antiguo-, después como una continua adaptación y remodelación de estas casas para albergar los usos y funciones propias de un edificio público de estas características. El edificio contiene todo aquello que a los arquitectos interesa. Por un lado, la presencia del tiempo en arquitectura, en un conjunto que, a la manera de un palimpsesto, alberga trazas, huellas y capas sucesivas de historia. Por otro, se trata de un conglomerado de piezas heterogéneas, cada una con lógica propia dentro del conjunto, yuxtapuestas en el tiempo, en la función y en el carácter.

De la planta anterior a la intervención de Miralles/Tagliabue destacan la secuencia de casas medievales paralelas al canal, culminadas por un envoltorio neoclásico envolviendo tres de las construcciones originales, además de un bloque rectangular en la parte posterior, el Registro Municipal, añadido en los años cuarenta del s.XX (Fig. 1). La propuesta de los arquitectos parte precisamente de la decisión de demoler este bloque para generar una nueva plaza urbana en el corazón de la ciudad en la que ubicar la entrada principal, un movimiento que resuelve en un solo gesto la demanda del concurso de dotar a la institución de un nuevo carácter arquitectónico ${ }^{3}$. Y es que si durante quinientos años de historia el Ayuntamiento ha vestido sus fachadas al canal del estilo más adecuado a cada época, desdibujando progresivamente la crujía de las construcciones originales y buscando un aspecto más unitario y monumental, en 1997 Miralles y Tagliabue proponen devolver al edificio el espíritu de la ciudad que lo vio nacer. En la memoria que meses después entregan a la prensa local, escriben: "Volver a la idea de un edificio municipal como un conglomerado de diferentes edificios urbanos"4.

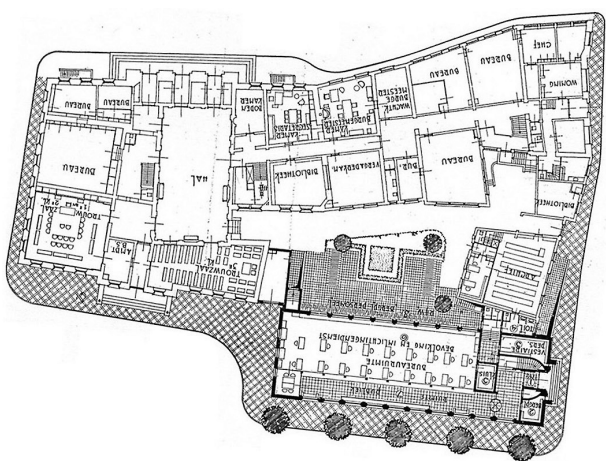

Fig. 1. Ayuntamiento de Utrecht. Planta baja. Estado 1941.

1. MORENO, Luis; TUÑÓN, Emilio,"Apuntes de una conversación informal [con Enric Miralles]", El Croquis 100/101, 2000 , p. 17.

2. FUTAGAWA, Yoshio, Studio Talk. Interview with 15 architects, A.D.A., Tokyo, 2002, p. 638.

3. Ver: JAMAR, Jo, The Town Hall of Utrecht, Uitgeverij Matriis, Utrecht, 2000; y De KAM, René, Een waardig huis der stad, Uitgeverij Matrijs, Utrecht, 2000.

4. MIRALLES/TAGLIABUE, The Project for the Town Hall of Utrecht. Document for the press representatives, Oct 1997, [Archivo Fundación Enric Miralles]. 


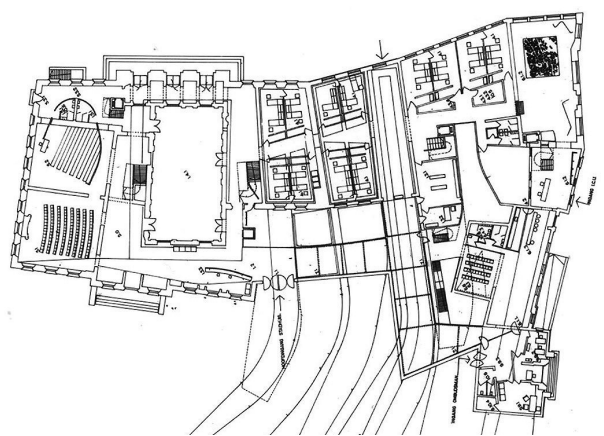

2 3

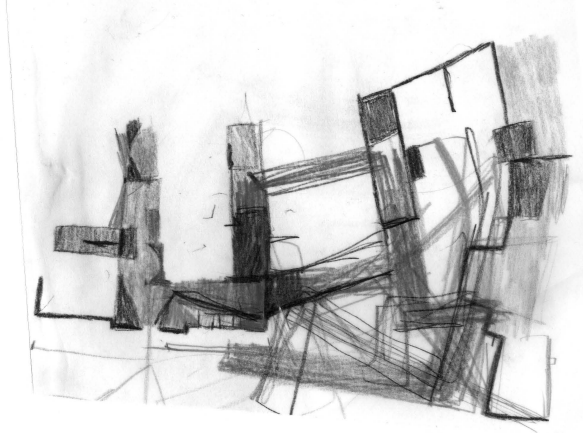

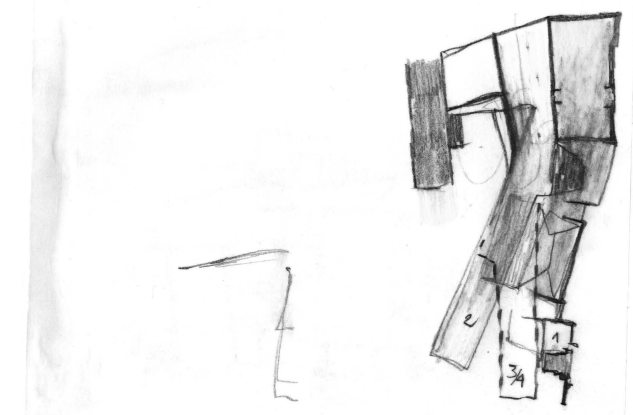

4
El Ayuntamiento se ha abierto a la ciudad. Lo que durante siglos había sido un conjunto de patios traseros es ahora la fachada principal del conjunto. Como se aprecia en la maqueta de concurso, desde las traseras de las construcciones medievales emergen unas piezas lineales de nueva construcción que mantienen la crujía de los edificios medievales, subrayando la idea de conjunto de casas que la propuesta quiere potenciar. Sin embargo, mientras que la propuesta urbana dota al edificio de un nuevo carácter de manera inmediata, difícilmente pueda decirse lo mismo de la planta del concurso, condicionada en gran parte por el esquema fragmentario y laberíntico del edificio original (Fig. 2). En la evolución del proyecto, la planta del Ayuntamiento adquiere un nuevo carácter esencialmente a través de la geometría, cuando el esquema compartimentado original es sustituido progresivamente por un trazado mucho más personal, vinculado a la manera de dibujar de Enric Miralles.

En el archivo de la Fundación Enric Miralles en Barcelona se conservan 479 dibujos originales de Enric Miralles para el proyecto de Utrecht ${ }^{5}$. La mayoría son croquis y planos de planta elaborados en papel vegetal, cuyo tamaño oscila aproximadamente entre el DIN A5 y el DIN A3, principalmente a escala $1 / 400$ y 1/200. Entre el concurso y la solución finalmente construida median cuatro versiones intermedias. Los sucesivos cambios en el proyecto desde febrero de 1997 a la versión final de octubre de 1999 estarán influenciados por dos aspectos fundamentales, que Miralles controla desde la planta: primero, cómo establecer la relación con la nueva plaza e insertar la nueva arquitectura en el vacío dejado tras la demolición del bloque del Registro; segundo, cómo dotar al edificio de una estructura lógica de circulaciones y programa, a pesar de la fragmentación impuesta por una sección en la que a las distintas construcciones históricas corresponden distintas cotas de altimetría.

Siendo Utrecht un proyecto tardío en la trayectoria de Miralles y Tagliabue, desarrollado en un momento en el que la oficina contaba con más de treinta colaboradores, la cantidad y minuciosidad de los planos archivados permiten identificar cuánto hasta el final Miralles siguió proyectando mediante dibujos propios que estructuraban el trabajo colectivo del estudio. Como recordará Benedetta Tagliabue años después: "Detrás de esa confusión organizada del estudio, la fuerza de los dibujos de Enric entraba en lid frente al vigor y la rapidez de esa maquinaria de treinta o cuarenta personas, deviniendo en una producción sorprendente" ${ }^{\text {. }}$

Cuando Enric Miralles, a través de dibujos sucesivos, comienza a buscar una nueva estructura para el Ayuntamiento de Utrecht, se produce una incorporación de su manera de dibujar a la geometría de la planta. La evolución del proyecto muestra una diferenciación progresiva respecto del carácter arquitectónico del edificio original. No obstante, las primeras versiones son todavía deudoras del esquema medieval, mostrando cómo la propuesta se piensa esencialmente desde la estrategia urbana, pero cuánto la distribución interior hereda la estructura fragmentaria de la planta histórica. Bien es cierto que se producen cambios de carácter arquitectónico, impulsados sobre todo por la demolición del bloque del Registro y la creación del espacio público de la nueva plaza en su lugar, pero la manera de entretejer nuevo y viejo es igual a la que había caracterizado la historia del edificio, mostrando un código geométrico que hereda las medidas de crujía de las casas medievales y que se construye en base a circulaciones rectas y quebradas, dando lugar a un conglomerado de espacios y habitaciones difícilmente relacionables entre sí.
5. En enero de 2010, durante el desarrollo de la tesis doctoral, el autor tuvo acceso a todos los dibujos de Enric Miralles para el proyecto del Ayuntamiento de Utrecht, gracias a colaboración de Benedetta Tagliabue. Asimismo, llevó a personalmente la digitalización de los mismos en el estudio EMBT en Barcelona.

6. TAGLIABUE, Benedetta, "Familias", en El Croquis 100/101 2000 , p. 24. 


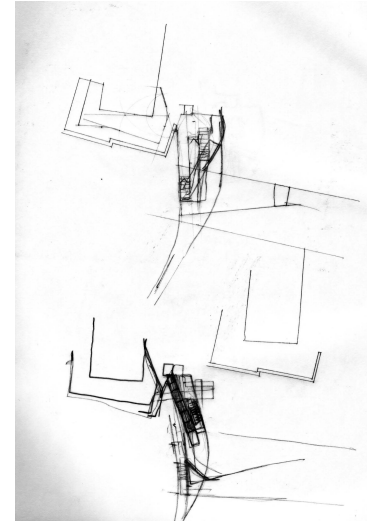

5

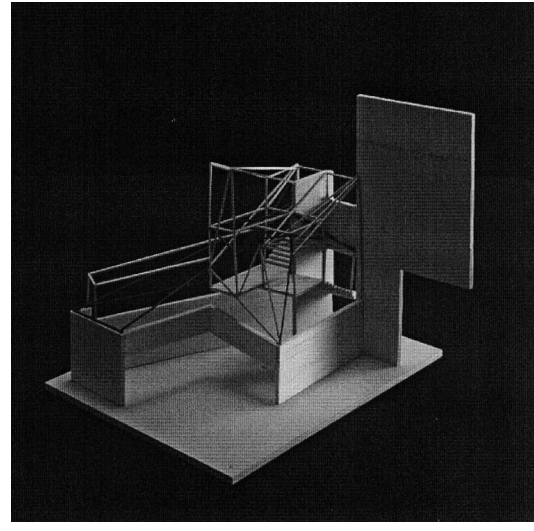

6

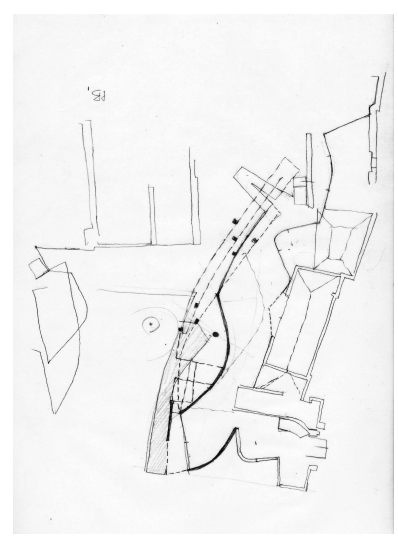

7

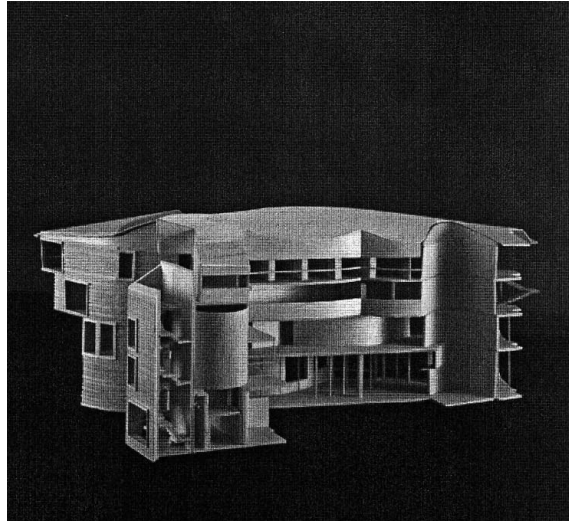

Es decir, la demolición de algunos edificios no previene al Ayuntamiento de seguir siendo una colección de arquitecturas dispersas, mostrando las primeras versiones del proyecto gran ambigüedad en la manera de integrar las nuevas construcciones en la mitad demolida de las casas medievales, y sobre todo en el frente hacia la nueva plaza, donde una sala lineal de exposiciones en el frente hacia la plaza en planta baja no resuelve con claridad la relación del edificio con el espacio público exterior y la dinámica de circulaciones interiores. La serie de dibujos sobre este tema en mayo, elaborados sobre todo en pequeños croquis a 1/400 y trazos gruesos con color para diferenciar distintos programas, muestra las dudas de Miralles en el proceso creativo.

Miralles produce esencialmente dos familias de dibujos: una en la que prueba la estructura general de toda la planta (Fig. 3), y otra en la que se centra en una parte del proyecto, los bloques de nueva inserción que se adosan a las casas de la calle lateral, formando un conglomerado que denomina "el nuevo ala"7 (Fig. 4). Respecto a la configuración general de la planta, Miralles dibuja una y otra vez el pabellón lineal de exposiciones en el frente de la plaza, poniéndolo en relación con el interior del edificio y tratando de estructurar a través de esta relación la geometría de la base del proyecto. Le preocupa la conexión de los distintos programas públicos en planta baja, así como la relación que establecen estos usos con las piezas de nueva construcción en las plantas superiores. A pesar de no haber un código gráfico claro, es fácil reconocer cómo los colores diferencian las zonas de circulación libre respecto de los elementos que la obstaculizan. Hay una intención clara en todos estos dibujos de escapar del orden compartimentado de las casas medievales y de dotar a la planta de una nueva configuración, a través de una mayor fluidez en los recorridos. A Miralles no le interesa la estructura fragmentaria original y por eso vuelca las circulaciones hacia la nueva plaza, donde hay un ámbito de movimientos libre.

Pero a partir de agosto Miralles y Tagliabue identifican la configuración de la planta para el Ayuntamiento de Utrecht. Eliminan la sala de exposiciones como una construcción añadida, lo que permite leer la planta del edificio como un gesto unitario en todos sus niveles, una herradura de brazos asimétricos en cuyos laterales se adosan dos piezas de nueva inserción: el pabellón de la entrada principal a la izquierda y el nuevo ala de oficinas a la derecha. Estas categorías, arquitectónicas, se corresponden además con una estructura de representación, con una particular manera de dibujar y pensar la arquitectura. El gesto unitario en herradura se define en plantas de conjunto, mientras que las piezas de nueva inserción se desarrollan en plantas y maquetas independientes, perdiendo cualquier analogía formal con las casas medievales (Figs. 5 a 8). La fragmentación de la representación y de la arquitectura misma desde la fragmentación de la planta es evidente. Se define así un sistema de trabajo en el que Miralles dibuja contornos en planta sobre los que se van probando distintas configuraciones tridimensionales en maqueta. La maqueta acompaña el proceso de trabajo de principio a fin, desde los primeros croquis a los planos finales, identificándose maquetas pequeñas y unitarias en las primeras versiones de proyecto, grandes y fragmentadas en las finales.

Miralles identifica en sus dibujos que la forma del gesto unitario en herradura tiene que emplear un sistema geométrico diferente al de la planta histórica: las líneas de distribución empiezan entonces a curvarse y plegarse sucesivamente, incorporando la energía de su ma-
Fig. 2. Miralles/Tagliabue: Remodelación del Ayuntamiento de Utrecht. Propuesta de concurso. Planta baja. Febrero 1997.

Fig. 3. Enric Miralles: Remodelación del Ayuntamiento de Utrecht. Croquis de la estructura general de la planta. Mayo 1997. [e: $1 / 400,21 \times 17 \mathrm{~cm}$ ].

Fig. 4. Enric Miralles: Remodelación del Ayuntamiento de Utrecht. Planta parcial de configuración del nuevo ala. Mayo 1997. [e: $1 / 400,21 \times 15 \mathrm{~cm}$ ].

Fig. 5. Enric Miralles: Remodelación del Ayuntamiento de Utrecht. Planta parcial del pabellón de la entrada principal. Octubre 1997. [e: $1 / 400,20 \times 29 \mathrm{~cm}$ ]

Fig. 6. Miralles/Tagliabue: Remodelación del Ayuntamiento de Utrecht. Maqueta parcial del pabellón de la entrada principal. Octubre 1997.

Fig. 7. Enric Miralles: Remodelación del Ayuntamiento de Utrecht. Planta parcial del nuevo ala. Octubre 1997. [e: 1/200, $21.1 \times 29 \mathrm{~cm}]$

Fig. 8. Miralles/Tagliabue: Remodelación del Ayuntamiento de Utrecht. Maqueta parcial del nuevo ala. Octubre 1997. 


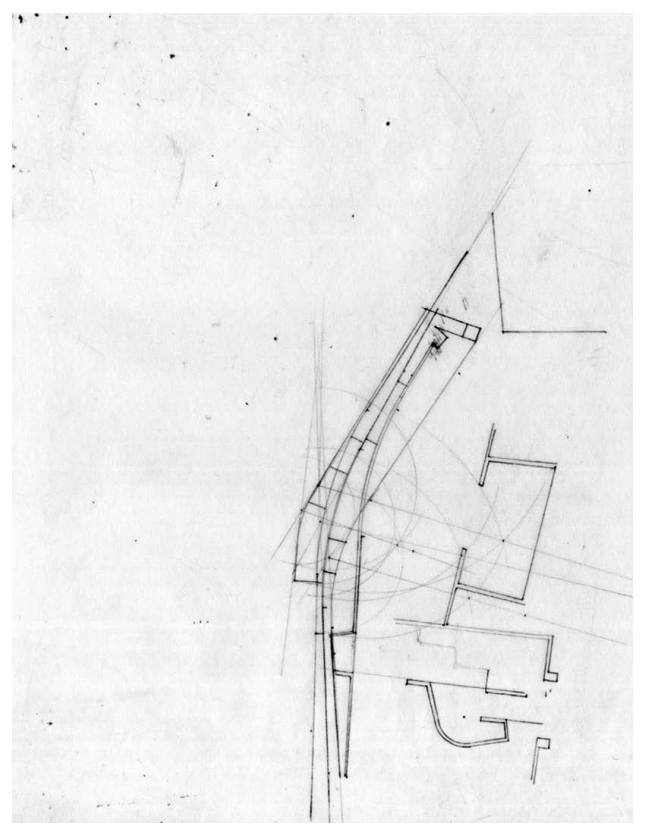

9
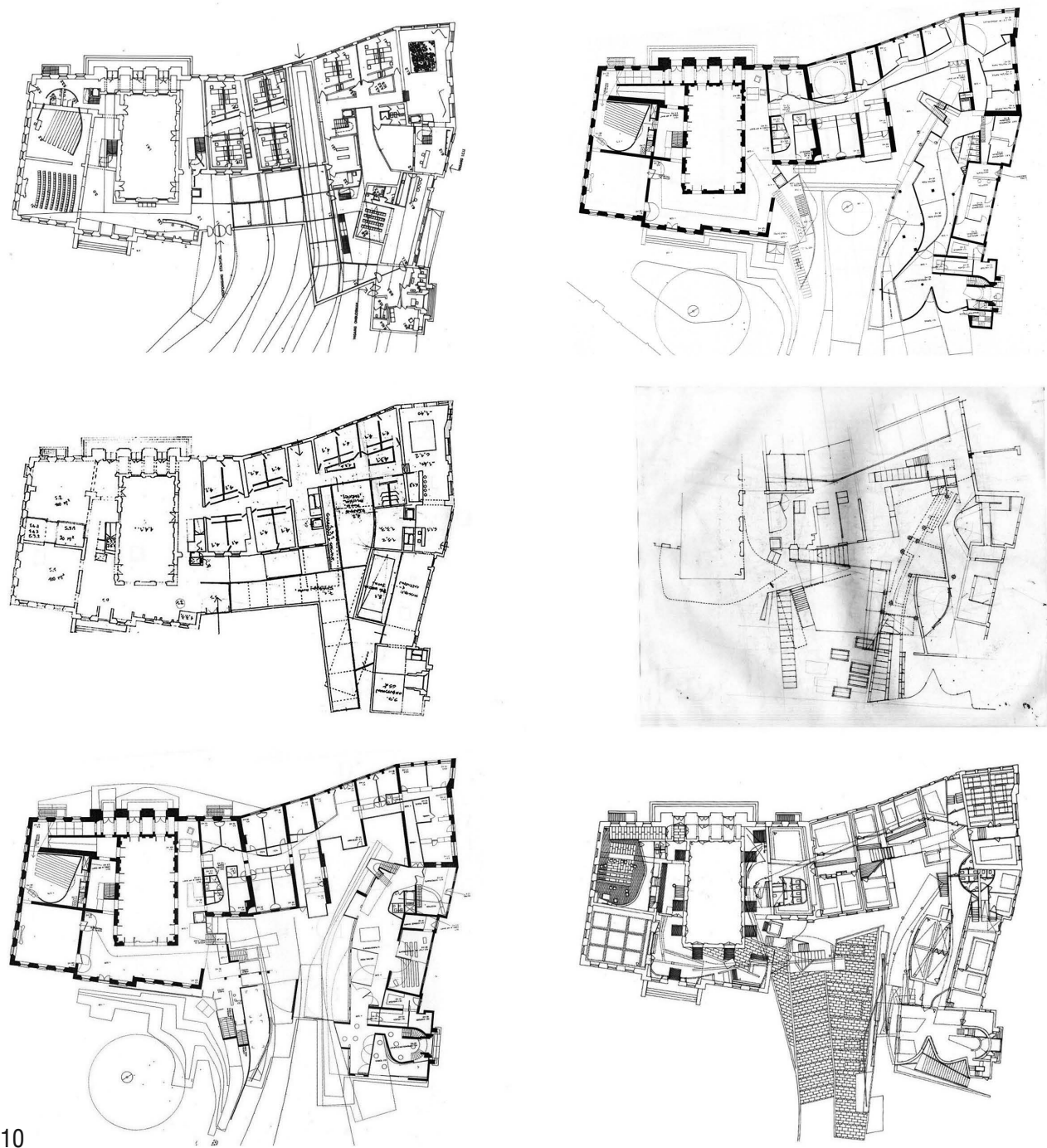

nera de dibujar al plano de arquitectura. Es importante subrayar cuánto a través de dibujos sucesivos Miralles se deshace de la estructura agregada y confusa de la planta histórica para acoplarla a uno de sus tipos característicos en planta, la geometría en herradura que se observa en proyectos tan heterogéneos en programa, escala y carácter como la Iglesia en Roma, el IUA en Venecia o la Casa Damge, entre otros.

Fig. 10. Miralles/Tagliabue: Remodelación del Ayuntamiento de Utrecht. Visión conjunta de las seis versiones de proyecto. Columna izquierda, arriba-abajo: Febrero 1997, Mayo 1997 Agosto 1997 / Columna derecha, arriba-abajo: Octubre 1997 Diciembre 1997, Octubre 1998

8. Ver MONEO, Rafael, "On typology", en Oppositions 13 1978, pp. 23-45; y CURTIS, William, "Mapas mentales y paisajes sociales", en El Croquis 49/50, 1991, pp. 6-20.
Las familias geométricas en planta se convierten para Miralles en un hilo conductor de su trabajo, en el tipo arquitectónico no tanto vinculado a una razón programática o constructiva, sino más bien como un marco geométrico en el que la continuidad y el cambio se producen ${ }^{8}$. Y siempre vinculado a una idea de espacio que niega lo ortogonal, que se mueve en contra de toda concepción convencional de lo cúbico y lo estático desde el trazado geométrico en planta. En el caso de Utrecht, definido con precisión el esquema de trabajo, los planos se vuelven entonces incoloros, aumentan de escala hasta llegar a 1/200 y se dibujan en una oscilación continua entre la versatilidad del trazo con mina blanda y la precisión del dibujo técnico (Fig. 9). La consecuencia es clara: mientras que en las plantas del concurso y de mayo la nueva construcción es difícil de diferenciar de la fábrica vieja del edificio original, ahora estas diferencias son perfectamente reconocibles.

La visión conjunta de todas las versiones del proyecto permite dividirlas en el momento anterior y posterior a la identificación de esta estructura de trabajo, reconociéndose en las propuestas a partir de agosto un carácter arquitectónico semejante, debido a la manera de producir el encuentro entre el sistema geométrico de los muros medianeros originales y la caligrafía de Miralles (Fig. 10). Las versiones siguientes poco variarán de este esquema de trabajo: cambian la forma y el trazado de circulaciones, introduciéndose cambios motivados sobre todo por aspectos funcionales, como la manera de producir los accesos al sótano, la distribución del programa o 


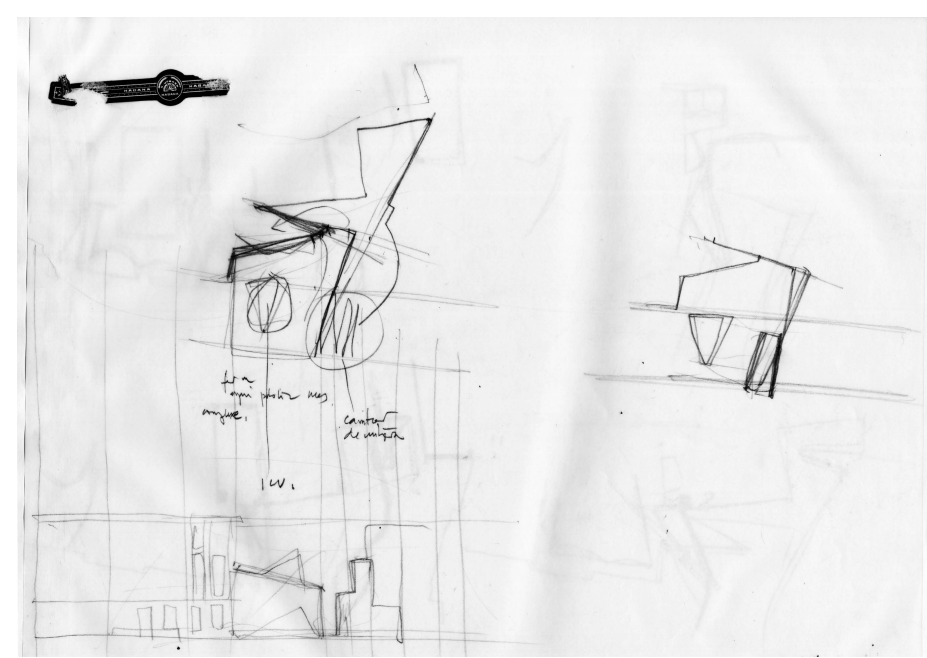

11

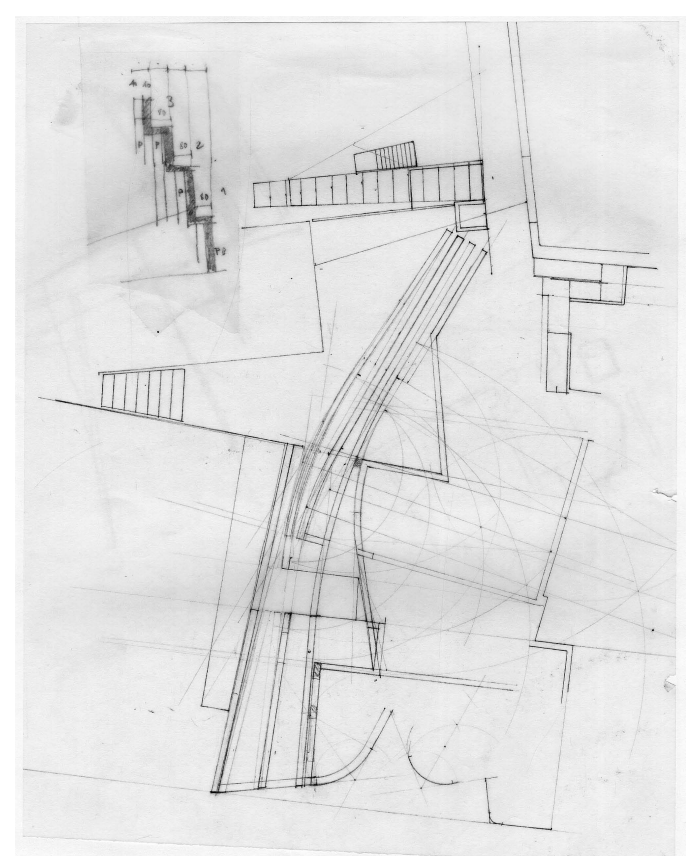

13

la relación entre el edificio y la plaza, pero el sistema de dibujo y pensamiento se mantiene. En todas las versiones habrá una planta general y plantas parciales, poniendo de manifiesto cuánto el cambio en una zona afecta al resto del edificio y viceversa, que en esta arquitectura no hay un pensamiento secuencial sino simultáneo de las partes y el todo. El cambio principal que experimenta el proyecto en diciembre está determinado por la 'presentación' del edificio hacia la plaza, sobre todo por la manera en que se produce el límite entre las caras traseras de las casas medievales y el espacio público, un límite para el que las versiones previas no habían encontrado una solución definitiva. En los primeros dibujos que Miralles hace antes de Navidad sobre este límite, redibuja la línea del mismo y la dota de grosor, buscando un borde más arquitectónico, con mayor presencia física, detrás del cual no haya sitio al que acceder. Para ello, rota la orientación del pabellón de la entrada principal, que se convierte en un cuerpo afilado vinculado a la línea de fondo de la plaza, y superpone una falsa fachada sobre el frente escalonado del nuevo ala, ocultando así parcialmente las circulaciones de servicio que alberga el foso.

Un pequeño croquis muestra la nueva estrategia urbana del proyecto (Fig. 11): en planta se reconoce el giro del pabellón de acceso y su vinculación al foso perimetral, mientras que en alzado se observa como el pabellón a la izquierda y la falsa fachada a la derecha completan la imagen del edificio hacia la plaza, el primero como una especie de navío encallado
Fig. 11. Enric Miralles: Remodelación del Ayuntamiento de Utrecht. Croquis de estrategia urbana del proyecto. Diciembre 1997. [e: $1 / 400,38 \times 29.7 \mathrm{~cm}]$.

Fig. 12. Enric Miralles: Remodelación del Ayuntamiento de Utrecht. Plano de planta primera.

Diciembre 1997. [e: 1/200, 37×29.2cm].

Fig. 13. Enric Miralles: Remodelación del Ayuntamiento de Utrecht. Planta parcial del nuevo ala. Plantas baja, primera, segunda y tercera superpuestas. Diciembre 1997. [e: $1 / 200,21 \times 26,8 \mathrm{~cm}$ ].

Fig. 14. Enric Miralles: Remodelación del Ayuntamiento de Utrecht. Croquis de la escalera de esquina. Diciembre 1997. [e:1/200, 36.7×29cm]. 


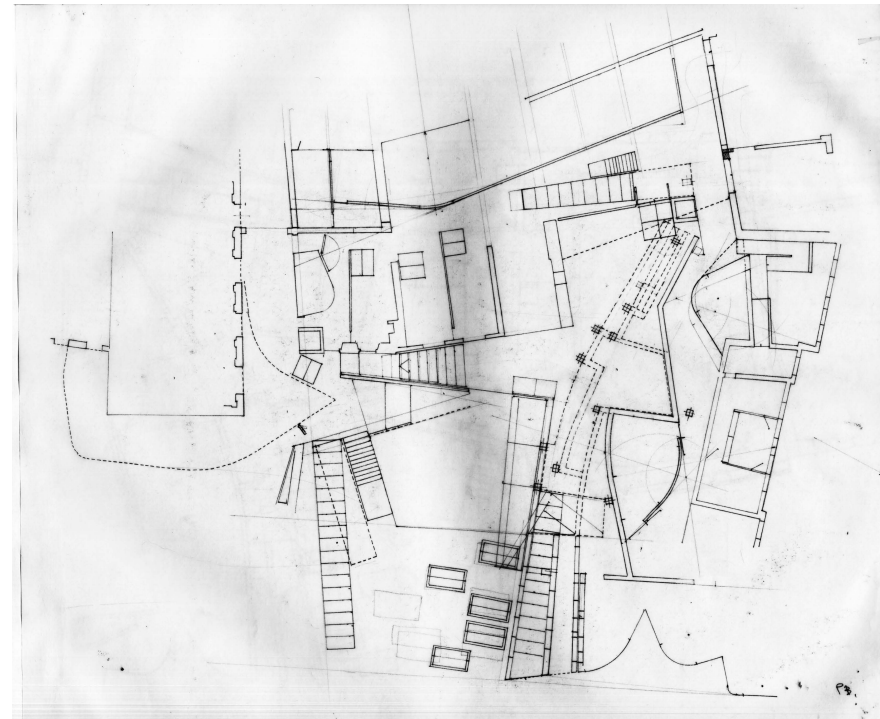

15

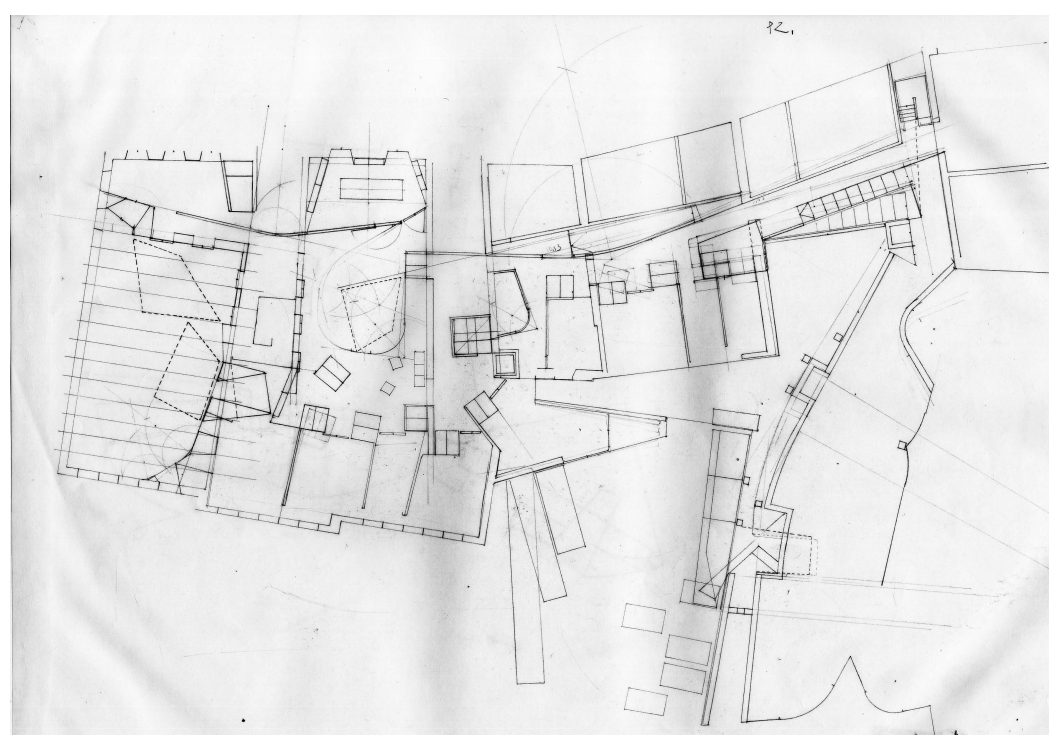

17

Fig. 15. Enric Miralles: Remodelación del Ayuntamiento de Utrecht. Plano de planta baja.

Diciembre 1997. [e: $1 / 200,35 \times 29.7 \mathrm{~cm}$ ].

Fig. 16. Enric Miralles: Remodelación del Ayuntamiento de Utrecht. Plano de planta primera.

Diciembre 1997. [e: $1 / 200,39.2 \times 29.7 \mathrm{~cm}$ ].

Fig. 17. Enric Miralles: Remodelación del Ayuntamiento de Utrecht. Plano de planta segunda.

Diciembre 1997. [e: $1 / 200,40.6 \times 29.7 \mathrm{~cm}$ ].

Fig. 18. Enric Miralles: Remodelación del Ayuntamiento de Utrecht. Plano de planta tercera.

Diciembre 1997. [e: $1 / 200,33.8 \times 29.1 \mathrm{~cm}$ ]

16
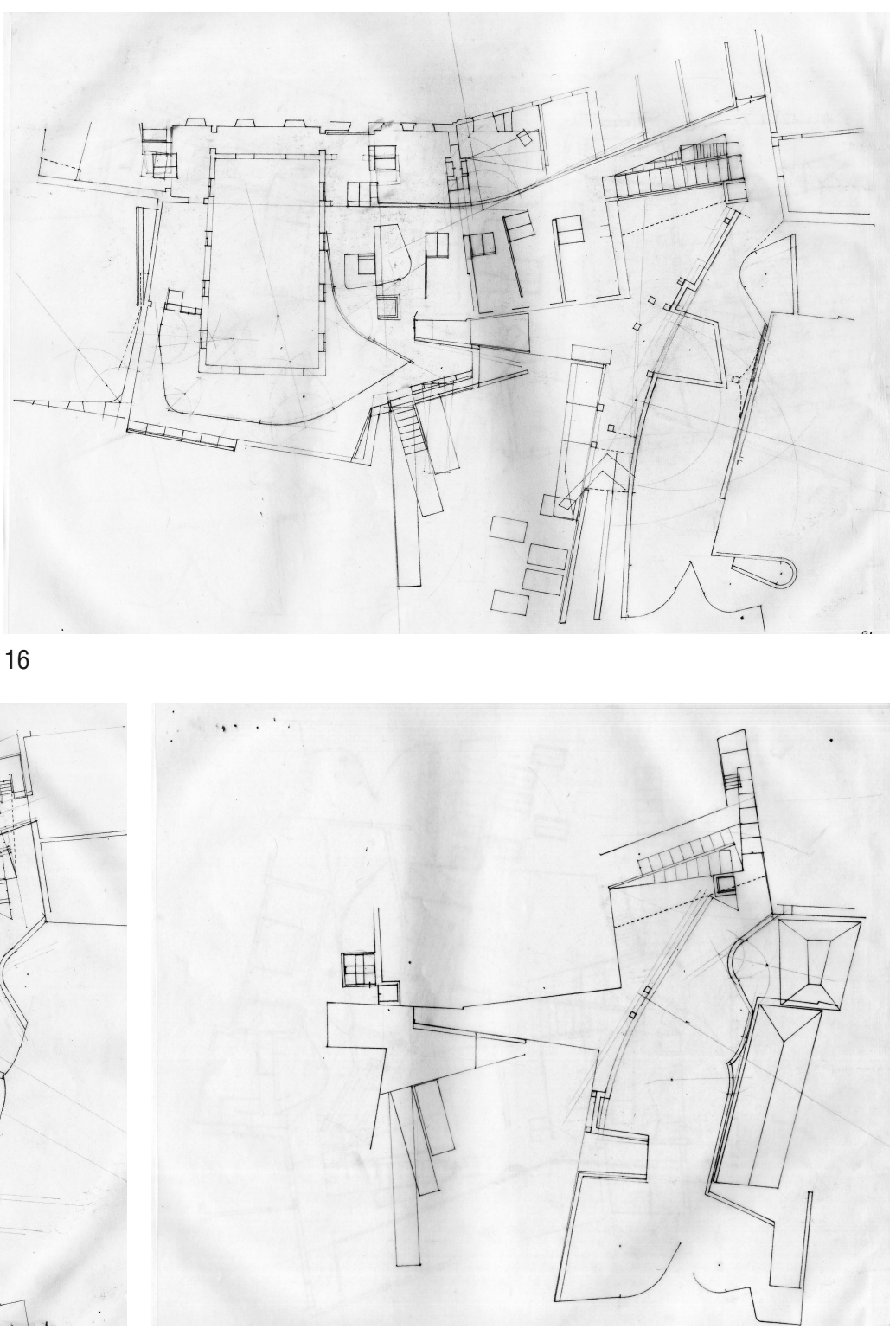

18

contra el testero del bloque neoclásico, la segunda escalonándose de forma plástica y buscando una cierta resonancia con aquello que oculta parcialmente. Uno y otro, sometidos a una particular evolución, se mantendrían hasta el final del proyecto.

Decidida la presencia urbana del edificio, Miralles y Tagliabue se centran en resolver la estructura interior de la planta, en hacer que funcione, más allá de las piezas que la componen, como una unidad. Miralles produce entonces una serie extraordinaria de planos a escala 1/200 en DIN A3, donde representa esencialmente las líneas que constituyen la geometría soporte del proyecto. Por un lado trabaja con plantas de conjunto (Fig. 12), desglosadas en calcos sucesivos debido al aumento de detalle de los planos, y por otro con plantas parciales del nuevo ala y de la escalera que articula la junta entre esta construcción y las casas hacia el canal (Figs. 13 y 14).

En las plantas definitivas de la versión de diciembre, se reconoce la vinculación geométrica entre el pabellón de entrada y el nuevo ala (Figs. 15 a 18). A pesar de la independencia formal que ambos cuerpos habían mostrado en la evolución previa del proyecto, los planos de esta versión presentan una gran atención a la relación de ambos en planta, alineándose la parte interior del primero con los recortes en el segundo, algo que condiciona su distribución funcional. En toda la serie de dibujos de esta fase del proyecto no se conservan croquis de 
Miralles en sección. Como recordaran importantes colaboradores del proyecto en distintas fases del mismo, Miralles solía delegar en ellos el trabajo sobre la sección, bien a través de planos o maquetas, dando indicaciones e instrucciones al respecto, pero sin mostrar la misma preocupación por la definición personal de la geometría a través de su manera de dibujar9

De marzo a octubre de 1998 se elabora el proyecto de ejecución del Ayuntamiento de Utrecht, extendiéndose el diseño del entorno urbano hasta octubre de 1999. Resueltos los problemas relativos a las circulaciones interiores, las modificaciones introducidas en la versión definitiva se refieren sobre todo a la relación del edificio con la nueva plaza y a la geometría del pabellón de entrada y del nuevo ala. La secuencia de trabajo es idéntica a la de fases anteriores: planta general de la estructura unitaria del proyecto, y plantas parciales de las piezas de nueva construcción. Finalmente el pabellón de la entrada principal se construye como un recorrido que sale del edificio para volver a entrar en él, combinando dos tramos de circulaciones, el primero constituido por una escalera que arranca desde la entrada, el segundo por una pasarela que devuelve al visitante al espacio interior. Mantiene el ancho variable de la escalera pero cambia la figura trapezoidal por una geometría similar a la de algunos elementos del proyecto del Parlamento de Edimburgo, basados en motivos orgánicos como hojas o barcas en planta (Fig. 19). El concurso del Parlamento se gana en junio, mientras que los dibujos de este pabellón para Utrecht son del mes de julio, por lo que es fácil imaginar que de un proyecto a otro se produjeran transferencias geométricas.

En paralelo a todos estos planos Miralles desarrolla otros detalles, como muestra de un proceso de trabajo que se acerca a su final. Mientras que las versiones precedentes estaban muy centradas en la definición de la forma del espacio, ésta también lo estará en la definición de su materialidad. Sin embargo, no habrá en este apartado un desarrollo tan extenso ni tan detallado como en los planos de planta. Miralles producirá en lo referente a la construcción y acabados dibujos de instrucciones, croquis llenos de anotaciones sobre los materiales o la forma misma, pero sin llegar a la obsesión por la definición precisa de la geometría que había caracterizado el desarrollo previo del proyecto. Se trata sobre todo de dibujos que permiten definir los interiores del edificio, combinándolos con las preexistencias históricas del mismo. Como mostraría la obra construida, Miralles y Tagliabue producen en muchos casos un trabajo arqueológico, decidiendo qué capas del pasado ocultan y cuáles activan y dejan visibles, similar al que ya habían llevado a cabo en su casa en la Calle Mercaders.

Miralles elabora también dibujos de otros elementos como puertas, bancos o sillas, que se convierten en esta arquitectura en objetos con valor en sí mismo, más allá de sus funciones primigenias, adquiriendo en muchos casos significados y funciones añadidas: cinéticas, escultóricas, etc. Como muestra el croquis de la puerta de entrada al aparcamiento de bicicletas, en estos dibujos se mezclan las escalas y proyecciones necesarias para definir el objeto arquitectónico con anotaciones referentes a los materiales y a la construcción misma (Fig. 20).

En los planos de la propuesta final se reconoce mayor densidad de información respecto a versiones precedentes. Aspectos como los pavimentos o los acabados ayudan a vivificar unos interiores hasta entonces desnudos. En las plantas destaca la estructura unitaria del conjunto, conseguida mediante la fluidez y suavidad de unas circulaciones interiores en las que la línea de pasillos corta de manera indiferente los muros medianeros de la fábrica original. La versión final del Ayuntamiento muestra por tanto un delicado encaje entre viejo y nuevo, donde las piezas de nueva construcción, pabellón de entrada y nuevo ala, se acoplan perfectamente con el edificio preexistente, y donde las intervenciones sobre la fábrica vieja del conjunto se muestran como una capa más superpuesta a la larga historia del edificio.

La planta del Ayuntamiento pasa así de tener una estructura construida por la historia a tener una configuración definida por una manera de dibujar. Como hemos visto, Miralles dibuja (y piensa): a mano, en papel vegetal que superpone sobre la versión anterior de la planta, en escalas pequeñas cuando busca el gesto unitario del conjunto, en aumentos progresivos de escala y fragmentación de la planta para definir con precisión la geometría del proyecto y de sus piezas constituyentes. La repetición de este proceso en el tiempo produce una presencia manual en la planta de Utrecht. Enric Miralles solía referirse con frecuencia al

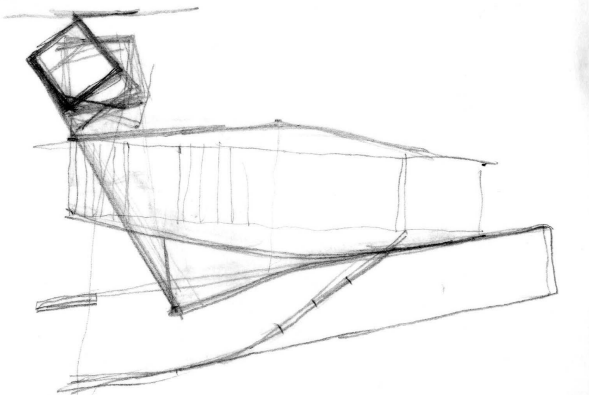

Fig. 19. Enric Miralles: Remodelación del Ayuntamiento de Utrecht. Planta parcial del pabellón de la entrada principal. Julio 1998. [e: $1 / 100,21.6 \times 16.4 \mathrm{~cm}$ ]

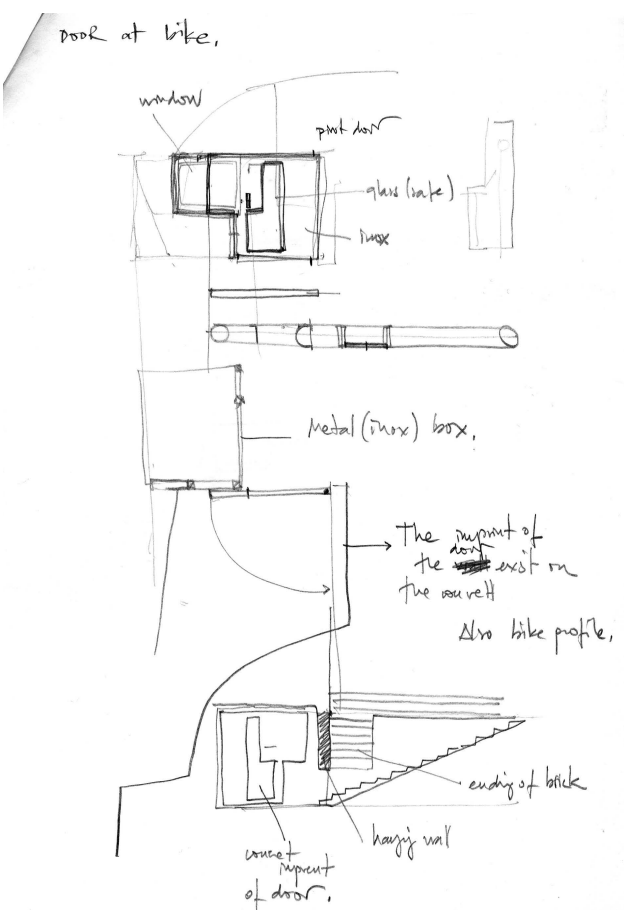

Fig. 20. Enric Miralles: Remodelación del Ayuntamiento de Utrecht. Planos de detalle de acceso al parking de bicicletas. Marzo 1998. [21x29.7cm].

9. Información facilitada por Ricardo Flores, antiguo colaborador del estudio EMBT y director del proyecto del Ayuntamiento de Utrecht en la primera fase de su desarrollo, [Entrevista realizada por el autor, Barcelona, 29-05-2010]. 
10. MIRALLES, Enric. Cosas vistas a izquierda y derecha (sin gafas). Tesis doctoral. 1987. Primera versión y anexo conservados en tres volúmenes en la Biblioteca del COAC. Códigos: D-27.316, D-27.317, D-27.318. Segunda versión conservada en tres volúmenes en la Biblioteca de la ETSAB. Códigos: RT Miralles Reg. 24.485, R-T-Miralles Reg. 24.486.

11. Para una descripción de las vicisitudes del proceso académico de la tesis doctoral de Enric Miralles, ver: MONEO, Rafael, "Cosas vistas a izquierda y derecha (sin gafas). Un comentario sobre la tesis doctoral de Enric Miralles Moya", en $D C$ Revista del Departamento de Composición Arquitectónica ETSAB, n. 17-18, febrero 2009, pp. 96-108

12. MIRALLES, E., Cosas vistas a izquierda y derecha (sin gafas), Tesis doctoral, 1987, D-27.317, p. 5.

13. MIRALLES, E., "Un retrato de Giacometti", en El Croquis, 72[II], 1995, pp. 129-132.

14. MIRALLES, E., "La répétition, la différence", en L'Architecture d'Aujourd'hui 312, Enric Miralles - un potrait, 1997 pp. 76-77.

15. "La repetición es muy importante porque cada nuevo dibujo efectúa una operación de olvido, y las leyes que se van generando son de coherencia interna". En: ZAERA, Alejandro, "Una conversación con Enric Miralles", en El Croquis 72[II] 1995, p. 14. concepto de 'repetición' como definidor de su sistema de trabajo, una idea prestada y adaptada de manera muy personal del libro ‘Diferencia y Repetición' de Gilles Deleuze, sólo citado de forma explícita en su tesis doctoral 'Cosas vistas a izquierda y derecha'10, pero implícito en sus reflexiones en numerosos escritos y entrevistas.

Presentada por primera vez en noviembre de 1987 en dos escuetos volúmenes ${ }^{11}$, la tesis establece la discusión en torno a la relación entre anotación y pensamiento en los cuadernos de los viajeros del Grand Tour. En ella Miralles se centra en encontrar una forma de pensamiento que se entremezcle con lo gráfico. "En este trabajo este pensamiento lo veremos como el eco de tres actores: ojo, trazo, papel" 12 , escribe al principio. Si el pensamiento gráfico habla de un orden físico, en el sentido de implicación de la mano y del ojo en el acto de pensar, la repetición habla de un orden temporal, siendo la deuda intelectual con Deleuze evidente. Aparentemente antagónicos, los conceptos de diferencia y repetición están indisolublemente unidos en su pensamiento. En la tesis, Miralles los personaliza y emplea desde una doble dimensión social e individual: por un lado, los viajeros en su conjunto, que generación tras generación repiten el mismo viaje produciendo siempre visiones distintas; por otro, el viajero como individuo, que no devuelve la misma interpretación del hecho observado en dos momentos diferentes de su vida.

Como concepto, la repetición habla de cómo un mismo pensamiento, por el hecho de producirse, de "hacerse físico" en dos instantes distintos, incorpora variaciones. Por tanto, no se trata tanto de mantener las propiedades de las ideas en las formas, ni de buscar la diferencia como una cualidad en sí misma, sino de admitir literalmente que la misma idea formaliza$d a$ en dos instantes diferentes no es igual. La repetición no es mímesis. Hay que distinguir claramente entre lo que se repite -las ideas- y lo que cambia -su actualización física-. La referencia por parte de Miralles a este concepto y a su importancia en el proceso creativo será una constante a lo largo de su carrera. Los escritos Un retrato de Giacometti ${ }^{13}$ o La repetition, la difference ${ }^{14}$, entre otros, insistirán en esta idea y darán claves para entender su obra y pensamiento. En efecto, la noción de que la repetición de una misma idea en momentos diversos produce diferencias le servirá para explicar la vinculación de su personal geometría a la ejecución de múltiples dibujos en la secuencia de trabajo de un mismo proyecto, como mecanismo para desprenderse de los condicionantes y limitaciones de partida. Forma y geometría vinculadas por tanto a la repetición del dibujo en el tiempo. Un método que Miralles resumió en la frase "cada nuevo dibujo efectúa una operación de olvido" 15 y, efectivamente, en cada una de las propuestas para Utrecht se produce un olvido progresivo de las constricciones de la planta original, que conduce a la diferencia e identidad del edificio final.
Javier Fernández Contreras (Granada, 1982). Es Arquitecto por la Escuela Técnica Superior de Arquitectura de Madrid (ETSAM) desde 2006, Doctor Internacional en Proyectos Arquitectónicos Avanzados por la ETSAM en 2013 con la tesis doctoral 'La Planta Miralles: Representación y Pensamiento en la Arquitectura de Enric Miralles', finalista en el X Concurso Bienal de Tesis de Arquitectura Arquia/Tesis 2015. Su trabajo e investigación han sido publicados en diversas revistas y libros especializados, como Massilia Annuaire d'études corbuséennes, Revista Arquitectura, CIRCO, 306090, Perspectives in Metropolitan Research. Ha sido Profesor Investigador en Formación en el Departamento de Proyectos de la ETSAM, Profesor de Proyectos Arquitectónicos en XJTLU en China, Profesor Ayudante de Proyectos Arquitectónicos en ETH Zurich. 